\title{
FIRST-ORDER LOGIC FOUNDATION OF RELATIVITY THEORIES
}

\author{
JUDIT X. MADARÁSZ, ISTVÁN NÉMETI AND GERGELY SZÉKELY
}

\begin{abstract}
Motivation and perspective for an exciting new research direction interconnecting logic, spacetime theory, relativityincluding such revolutionary areas as black hole physics, relativistic computers, new cosmology — are presented in this paper. We would like to invite the logician reader to take part in this grand enterprise of the new century. Besides general perspective and motivation, we present initial results in this direction.
\end{abstract}

\section{Introduction (LOGic AND SPACETIMe GeOMEtry)}

Throughout their intimately intertwined histories, logic and geometry immensely profited from their interactions. In particular, logic greatly profited from its applications to geometry. Indeed, the very birth of logic was brought about by the needs of geometry in the times of Socrates, Euclid and their predecessors. Ever since, their interactions had rejuvenating, invigorating effects on logic. For brevity, here we mention only Hilbert's axiomatization of geometry, Tarski's improvements on this in the framework of first-order logic (FOL) [62, Tarski's school of FOL approaches to geometry as a small sample. It is no coincidence that Tarskian algebraic logic is geometrical in spirit.

In this paper we try to show that this fruitful cooperation promises new blessings for logic. This is so because there are breathtaking revolutions in our understanding of space and time, i.e. in relativity, cosmology, and black hole physics.

What is the subject matter of geometry? Traditionally, geometry was created as a mathematical theory of a physical entity called space. But recent developments in spacetime theory/general relativity show that there is no such thing as physical space. Space is only an illusion and as such is subjective. Space is a "slice" of a larger entity called spacetime. Spacetime, on the other hand, is objective, it exists. What is subjective about space is the, necessarily ad hoc, way we decide to "slice" spacetime up into spacelike slices. Actually, it was logician Kurt Gödel who first discovered and emphasized that in certain non-negligible cases such slicing is impossible (non-foliazibility, in the technical terminology) [25.

Date: 2006. Apr. 10. 
So, a great challenge for logic and logicians is to continue the tradition sketched above of providing foundation and conceptual analysis for geometry by doing the same to spacetime theory, hence to relativity.

A further motivation for geometry-friendly logicians is the following. Relativity theory can be conceived of geometrizing parts of physics in a sense, cf. 46. Special relativity (SR) geometrizes some basic aspects of motion (kinematics) including light propagation; general relativity (GR) geometrizes gravitation + SR; the Kaluza-Klein style extension of GR geometrizes electromagnetic phenomena + GR; and currently intensively researched extensions of GR (e.g. string theory) search for extending the scope of this aim for geometrizing more and more aspects of our understanding of the world.

Why is this interesting for logicians? Well, because history tells us that logic is applicable to geometry in an essential way. Hence if relativity (and its extensions) is the act of geometrizing more and more of physics, then it also can be regarded as a potential act of "logicizing" these areas, inviting logicians to take part in this grandiose adventure of mankind.

\section{More Concrete introduction (FOUndation of SPACEtime)}

The idea of elaborating the foundational analysis of the logical structure of spacetime theory and relativity theories (foundation of relativity) in a spirit analogous with the rather successful foundation of mathematics was initiated by several authors including David Hilbert 34, cf. also Hilbert's 6th problem [33, Patrick Suppes [59], Alfred Tarski 32] and leading contemporary logician Harvey Friedman [22, 23.

There are several reasons for seeking an axiomatic foundation of a physical theory [60. One is that the theory may be better understood by providing a basis of explicit postulates for the theory. Another reason is that if we have an axiom system we can ask ourselves what axioms are responsible for which theorems. For more on this kind of foundational thinking called reverse mathematics, see for example, Friedman 22] and Simpson [56]. Furthermore, if we have an axiom system for special relativity or general relativity, we can ask what happens with the theory if we change one or more of the axioms. This could lead us to a new physically interesting theory. This is what happened with Euclid's axiom system for geometry when Bolyai and Lobachevsky altered the axiom of parallelism and discovered hyperbolic geometry.

Seeking a logical foundation for spacetime theory (i.e., roughly, relativity) is a worthwile attempt for several reasons. One of these is that spacetime can be regarded as a foundation of physics since spacetime is the arena in which physical phenomena take place. Another reason for seeking a logical foundation for spacetime is that throughout its history, logic benefited the most from those applications of logic which were aiming at branches of learning going through a turmoil or 
a revolutionary phase, and at the same time being important for our understanding of the world 35. As a quick glance to recent issues of, e.g., Scientific American can convince the reader, spacetime theory and relativity/cosmology certainly qualify. So we believe that it serves the best interest of logic community to apply logic to spacetime theory, relativity, cosmology, and black hole physics. Indeed, logic can benefit from such studies in many ways. As a bonus, as indicated in [14] or 38, spacetime theory can give a feedback to the foundation of mathematics itself.

For certain reasons, the foundation of mathematics has been carried through strictly within the framework of first-order logic (FOL). One of these reasons is that staying inside FOL helps us to avoid tacit assumptions. Another reason is that FOL has a complete inference system while higher-order logic cannot have one by Gödel's incompleteness theorem, see for example, Väänänen [65, p.505]. For more motivation for staying inside FOL as opposed to higher-order logic, see for example, [1], [2, Appendix 1: "Why exactly FOL"], 6], 21, 48, 67. The same reasons motivate the effort of keeping the foundation of spacetime and relativity theory inside FOL.

The interplay between logic and relativity theory goes back to around 1920 and has been playing a non-negligible role in works of researchers like Reichenbach, Carnap, Suppes, Ax, Szekeres, Malament, Walker, and of many other contemporaries. For more details, cf. e.g., 11. Also, it is no coincidence that relativity was the main motivating example for the logical positivists of the Vienna Circle.

Axiomatizations of SR have been quite extensively studied in the literature, see for example, the references of [1]. However, these works usually stop with a kind of representation theorem for their axiomatizations. As a contrast, what we call the foundation of relativity begins with the axiomatization (and representation theorems), but the real work and the real fun (the conceptual analysis) comes afterwards when we investigate, e.g., what axioms are responsible for which statements, what happens if we change the axioms etc.

While some FOL axiomatizations of the theory of inertial observers and for SR can be found in the literature ([6], 26], [1]), axiom systemslet alone FOL axiom systems - for accelerated observers and for GR are not too many in the literature (but cf. 44 for an exception).

In section 4, we recall a streamlined FOL axiomatization AccRel of SR extended with accelerated observers. In section [5], we take one step toward GR and investigate an aspect of time warp, that is the effect of gravitation on clocks, in our FOL setting. There we use Einstein's equivalence principle to talk about gravitation and prove the gravitational time dilation effect, that is that "gravity causes time to run slow", from AccRel in more than one sense. See Theorems 5.1, 5.2 and 5.3. We will also see that gravity can slow time down arbitrarily, see 
Theorems 5.4, 5.5 and 5.6. Furthermore, we investigate the role of the "direction" and the "magnitude" of gravitation in gravitational time dilation, see Theorems 5.7 and 5.8. We note that the most exotic features of black holes, wormholes and the like (mentioned in section 3 below) can be traced back to this effect of time warp (to be analyzed in section 5).

\section{Intriguing features of GR spacetimes (CHALlenges For THE LOGICIAN)}

Both SR and GR have many interesting consequences. Most of them show that we have to refine our common sense concepts of space and time. They are full of surprising predictions and paradoxes which seriously challenge our common sense picture of the world. But it is exactly this negation of common sense which makes this area an attractive field to apply logic.

Gravitation has many surprising effects on time. The common name for these effects is time warp.

For example, in the Schwarzschild spacetime, which is associated with a non-rotating black hole (or star), we face one of the simplest aspect of time warp called gravitational time dilation. There we see that if we suspend an observer closer to the black hole and another observer farther away from it, then the clock of the closer one will run slower than the clock of the one which is farther away. So in some sense we see that "gravity causes time run slow". There are places where this time warp effect becomes infinite, i.e. some clocks entirely stop ticking, i.e. freeze from the point of view of some other observers. Moreover, time and space may get interchanged. These effects are part of the reason why we said in section 1 that space does not exist while spacetime does.

The above-mentioned time warp effect leads to even stronger effects. We meet new interesting aspects of time warp in the Reissner-Nordström, Kerr and Kerr-Newman spacetimes that are associated with charged, rotating and charged-rotating black holes, respectively. For astronomical evidence for the existence of rotating black holes cf. e.g., 49], [58. In these spacetimes, there is an event whose causal past contains timelike curves which are infinitely long in the future direction. Such a curve can be the life-line of an observer (or computer) who has infinite time for working and sending light-signals that can be received before the distinguished event. The spacetimes in which these kinds of events occur are called Malament-Hogarth spacetimes, cf. e.g., Earman [15, §4], 38]. In Malament-Hogarth spacetimes, we can design a computer that decides non-Turing computable sets, cf. e.g., 38, 16, 14, 19]. Thus inside these spacetimes, we can decide whether an axiom system of set theory (for example $\mathrm{ZFC}$ ) is consistent or not. Therefore, in contrast with the consequence 
of Gödel's second incompleteness theorem, we can find out whether mathematics is consistent or not. For more detail on these kinds of computers in the physically reasonable Kerr spacetime, cf. e.g., [14, [19. Recently, the acceleration of the expansion of the universe made anti-de-Sitter spacetimes very popular with cosmologists. These also have the Malament-Hogarth property, hence are also suitable for harboring computers breaking the Turing barrier.

There are several models of GR in which there are so-called Closed Timelike Curves (CTC). Such are Gödel's rotating universe [25], Kerr and Kerr-Newman spacetimes [47, Gott's spacetime [27, Tipler's rotating cylinder 64, van Stockum's spacetime [57, Taub-NUT spacetime [31], to mention only a few. Since timelike curves correspond to possible life-lines of observers, in these spacetimes an observer can go through the same event more than once. This situation can be interpreted as time travel. This leads to non-trivial philosophical problems, in analysing/understanding which the methods of logic can considerably help. We believe, currently logic is the discipline best positioned for clarifying the apparent problems with CTC's, i.e. with time travel. Namely, the only problem with time travel is that it represents a kind of circularity, because of the following: a time traveler goes back into his past, changes his past so as to prevent his own existence, but then who went back into the past? etc. This circularity is not more vicious than the Liar paradox or self-reference implemented e.g. in Gödel's second incompleteness proof. Logic has been extremely successful in understanding and "de-mistifying" self-referential situations and the Liar paradox. Examples are provided by literature of Gödel's incompleteness method 30, the book on "The Liar" by Barwise and Etchemendy [7] which used non-well-founded set theory for providing an explicit semantic analysis for self-referential situations, [55. So logic seems to be best suited for providing rational understanding of situations like the circularity represented by CTC's or time travel. For more on CTC's, cf. e.g., [15, §6], [17, [28].

These are only a few of the many examples that show that turning Relativity Theory into a real FOL theory, axiomatizing it and analyzing its logical structure seem to be a promising, worthwile undertaking.

What could science gain from such a logical analysis of relativity theory? Turning GR into a FOL theory will make it more flexible. By flexibility we mean that we can change some of the axioms whenever we would like to change the theory, without having to re-build the whole theory from scratch. By changing the axioms, we can control the changes of theory better than by changing Einstein's field equations. This might be useful when we would like to understand the connection of GR to other theories of gravitation like the Brans-Dicke theory, cf. [8], 9], 20]. This flexibility can also be useful when we would like to extend GR. We indeed would like to extend GR since we do not 
have a good theory of Quantum Gravity (QG) which is a common extension of the quantum theory and GR. Some eminent researchers of relativity formulated an even more optimistic goal of searching for the geometrization of all physical phenomena known today into a so-called theory of everything (TOE). Of course, one wants both QG and TOE to be some kinds of extensions of GR.

Recent astronomical observations provided strong evidence that the expansion of our universe is accelerating. This discovery leads to many questions and to the idea that the cosmological constant might be replaced with a dynamical parameter, i.e. with a scalar field, called Quintessence or "dark energy" cf. e.g., [10, 13]. But this leads to a new need for modifying or at least fine-tuning GR. This also shows the merit of making GR more flexible by providing a FOL axiom system for it.

So far we have talked mainly about the significance of the logical foundation of GR, but the logical analysis of SR is also important since GR is built on SR. Moreover, there are other different relativity theories such as the Reichenbach-Grünbaum version, cf. [50], [51] and 29] or the Lorentz-Poincaré version of special relativity cf. 41. Their logical structures and connection with Einstein's relativity are also worth analyzing in order to get a more refined understanding of relativity theory. Our research group has done some work in this direction [2, $\S 4.5]$.

In the following sections we try to give a sample of the work done by our research group in Budapest in the direction of a FOL investigation of relativity theories (including GR).

\section{A FOL AXIOM System of SR EXtended With ACCELERATED OBSERVERS}

Here we recall one of our axiom systems for SR extended with accelerated observers (hence extended with a handle on gravity). We try to be as self contained as possible. First occurrences of concepts used in this work are set in boldface to make them easier to find.

The motivation for our choice of vocabulary is summarized as follows. Here we deal with the kinematics of relativity only, that is we deal with motion of bodies (or test-particles). We will represent motion as changing spatial location in time. To do so, we will have referenceframes for coordinatizing events and, for simplicity, we will associate reference-frames with special bodies which we will call observers. We visualize an observer-as-a-body as "sitting" in the origin of the space part of its reference-frame, or equivalently, "living" on the time-axis of the reference-frame. We will distinguish inertial observers from noninertial (accelerated) ones. There will be another special kind of bodies which we will call photons. For coordinatizing events, we will use an arbitrary ordered field in place of the field of the real numbers. Thus 
the elements of this field will be the "quantities" which we will use for marking time and space. Allowing arbitrary ordered fields in place of the field of the reals increases flexibility of our theory and minimizes the amount of our mathematical presuppositions, cf. e.g., Ax [6] for further motivation in this direction. Similar remarks apply to our flexibility oriented decisions below, for example, keeping the dimension of spacetime a variable. Using observers in place of coordinate systems or reference frames is only a matter of didactic convenience and visualization. Using observers (or coordinate systems, or reference-frames) instead of a single observer-independent spacetime structure has many reasons. One of them is that it helps us in weeding out unnecessary axioms from our theories; but we state and emphasize the equivalence/duality between observer-oriented and observer-independent approaches to relativity theory, cf. [42, §4.5]. Motivated by the above, we now turn to fixing the first-order language of our axiom systems.

We fix a natural number $d \geq 2$ for the dimension of spacetime. Our language contains the following non-logical symbols:

- unary relation symbols B (for Bodies), Ob (for Observers), $\mathrm{IOb}$ (for Inertial Observers), Ph (for Photons) and Q (for Quantities),

- binary function symbols + , · and a binary relation symbol $\leq$ (for the field operations and the ordering on Q), and

- a $2+d$-ary relation symbol W (for World-view relation).

The bodies will play the role of the "main characters" of our spacetime models and they will be "observed" (coordinatized using the quantities) by the observers. This observation will be coded by the worldview relation $\mathrm{W}$. Our bodies and observers are basically the same as the "test particles" and the "reference-frames", respectively, in some of the literature.

We read $\mathrm{B}(x), \mathrm{Ob}(x), \operatorname{IOb}(x), \operatorname{Ph}(x)$ and $\mathrm{Q}(x)$ as " $x$ is a body", " $x$ is an observer", " $x$ is an inertial observer", " $x$ is a photon", " $x$ is a quantity". We use the world-view relation W to talk about coordinatization, by reading $\mathrm{W}\left(x, y, z_{1}, \ldots, z_{d}\right)$ as "observer $x$ observes (or sees) body $y$ at coordinate point $\left\langle z_{1}, \ldots, z_{d}\right\rangle$ ". This kind of observation has no connection with seeing via photons, it simply means coordinatization.

$\mathrm{B}(x), \mathrm{Ob}(x), \operatorname{IOb}(x), \mathrm{Ph}(x), \mathrm{Q}(x), \mathrm{W}\left(x, y, z_{1}, \ldots, z_{d}\right), x=y$ and $x \leq y$ are the so-called atomic formulas of our first-order language, where $x, y, z_{1}, \ldots, z_{d}$ can be arbitrary variables or terms built up from variables by using the field-operations "+" and ".". The formulas of our first-order language are built up from these atomic formulas by using the logical connectives not $(\neg)$, and $(\wedge)$, or $(\vee)$, implies $(\Longrightarrow)$, if-and-only-if $(\Longleftrightarrow)$ and the quantifiers exists $x(\exists x)$ and for all $x(\forall x)$ for every variable $x$. 
The models of this language are of the form

$$
\mathfrak{M}=\langle U ; \mathrm{B}, \mathrm{Ob}, \mathrm{IOb}, \mathrm{Ph}, \mathrm{Q},+, \cdot, \leq, \mathrm{W}\rangle,
$$

where $U$ is a nonempty set and $\mathrm{B}, \mathrm{Ob}, \mathrm{IOb}, \mathrm{Ph}$ and $\mathrm{Q}$ are unary relations on $U$, etc. A unary relation on $U$ is just a subset of $U$. Thus we use $\mathrm{B}, \mathrm{Ob}$ etc. as sets as well, for example, we write $m \in \mathrm{Ob}$ in place of $\mathrm{Ob}(m)$.

$\mathrm{Q}^{d}:=\mathrm{Q} \times \ldots \times \mathrm{Q}(d$-times $)$ is the set of all $d$-tuples of elements of $\mathrm{Q}$. If $\vec{p} \in \mathrm{Q}^{d}$, then we assume that $\vec{p}=\left\langle p_{1}, \ldots, p_{d}\right\rangle$, i.e. $p_{i} \in \mathrm{Q}$ denotes the $i$-th component of the $d$-tuple $\vec{p}$. We write $\mathrm{W}(m, b, \vec{p})$ in place of $\mathrm{W}\left(m, b, p_{1}, \ldots, p_{d}\right)$, and we write $\forall \vec{p}$ in place of $\forall p_{1}, \ldots, p_{d}$ etc.

Let us begin formulating our axioms. We formulate each axiom at two levels. First we give an intuitive formulation, then we give a precise formalization using our logical notation (which easily can be translated into first-order formulas by substituting the definitions into the formalizations). We aspire to formulate easily understandable axioms in FOL.

The first axiom expresses our very basic assumptions like: both photons and observers are bodies, inertial observers are also observers, etc.

AxFrame: $\mathrm{Ob} \cup \mathrm{Ph} \subseteq \mathrm{B}, \mathrm{IOb} \subseteq \mathrm{Ob}, U=\mathrm{B} \cup \mathrm{Q}, \mathrm{B} \cap \mathrm{Q}=\emptyset$, $\mathrm{W} \subseteq \mathrm{Ob} \times \mathrm{B} \times \mathrm{Q}^{d},+$ and $\cdot$ are binary operations on $\mathrm{Q}, \leq$ is a binary relation on $\mathrm{Q}$.

To be able to add, multiply and compare measurements of observers, we put some algebraic structure on the set of quantities $Q$ by the next axiom.

AxEOF: A FOL axiom stating that the quantity part $\langle\mathrm{Q} ;+, \cdot, \leq\rangle$ is a Euclidean ${ }^{1}$ ordered field.

For the first-order definition of linearly ordered field, see for example, Chang-Keisler 11.

We will need some definitions to formulate our other axioms. Let $0,1,-, /, \sqrt{ }$ be the usual field operations which are definable from " + " and ".". We use the vector-space structure of $\mathrm{Q}^{d}$, i.e. if $\vec{p}, \vec{q} \in \mathrm{Q}^{d}$ and $\lambda \in \mathrm{Q}$, then $\vec{p}+\vec{q},-\vec{p}, \lambda \vec{p} \in \mathrm{Q}^{d}$; and $\vec{o}:=\langle 0, \ldots, 0\rangle$ denotes the origin. $\mathrm{Q}^{d}$ is called the coordinate system and its elements are referred to as coordinate points. We use the notation $\vec{p}_{s}:=\left\langle p_{2}, \ldots, p_{d}\right\rangle$ for the space component of $\vec{p}$ and $p_{t}:=p_{1}$ for the time component of $\vec{p} \in \mathrm{Q}^{d}$. The event (the set of bodies) observed by observer $m$ at coordinate point $\vec{p}$ is:

$$
e v_{m}(\vec{p}):=\{b \in \mathrm{B}: \mathrm{W}(m, b, \vec{p})\} .
$$

The coordinate-domain of observer $m$ is the set of coordinate points where $m$ observes something:

$$
C d_{m}:=\left\{\vec{p} \in \mathrm{Q}^{d}: e v_{m}(\vec{p}) \neq \emptyset\right\} .
$$

\footnotetext{
${ }^{1}$ That is a linearly ordered field in which positive elements have square roots.
} 


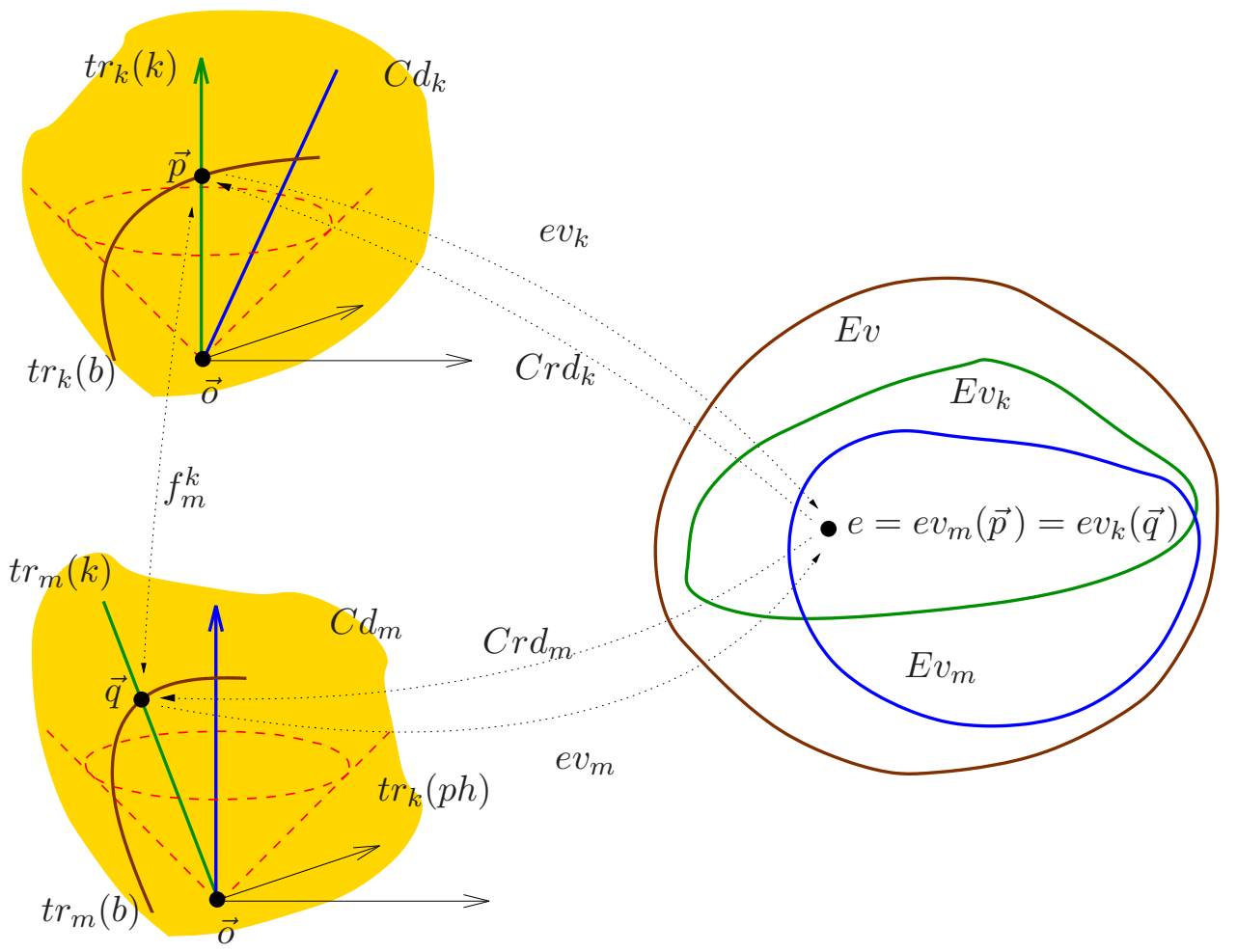

FiguRE 1. Illustration for the basic definitions.

Now we formulate our first axiom on observers. This natural axiom goes back to Galileo Galilei and even to d'Oresme of around 1350, cf. e.g., [1, p.23, §5]. It simply states that each observer thinks that he rests in the origin of the space part of his coordinate system.

AxSelf ${ }^{-}$: An observer sees himself in an event iff the space component of the coordinate of this event is the origin.

$$
\forall m \in \mathrm{Ob} \forall \vec{p} \in C d_{m} \quad\left(m \in e v_{m}(\vec{p}) \Longleftrightarrow \vec{p}_{s}=\vec{o}\right) .
$$

To formulate our axiom about the constancy of the speed of photons, for convenience, we choose 1 for this speed. Below, the Euclideanlength of $\vec{p} \in \mathrm{Q}^{n}$ is defined as $|\vec{p}|:=\sqrt{p_{1}^{2}+\ldots+p_{n}^{2}}$, for any $n \geq 1$.

$\mathrm{AxPh}_{0}$ : For every inertial observer, there is a photon through two coordinate points $\vec{p}$ and $\vec{q}$ iff the slope of $\vec{p}-\vec{q}$ is 1 :

$$
\begin{aligned}
\forall m \in \mathrm{IOb} \forall \vec{p}, \vec{q} \in \mathrm{Q}^{d}( & \left|\vec{p}_{s}-\overrightarrow{q_{s}}\right|=\left|p_{t}-q_{t}\right| \Longleftrightarrow \\
& \left.\operatorname{Ph} \cap e v_{m}(\vec{p}) \cap e v_{m}(\vec{q}) \neq \emptyset\right) .
\end{aligned}
$$

Motivations for this axiom can be found, for example, in [3], or in d'Inverno [12, §2.6].

The set of events seen by observer $m$ is:

$$
E v_{m}:=\left\{e v_{m}(\vec{p}): \vec{p} \in C d_{m}\right\},
$$


and the set of all events is

$$
E v:=\left\{e \in E v_{m}: m \in \mathrm{Ob}\right\} .
$$

With the next axiom, we assume that every inertial observer sees the same set of events.

AxEv: Every inertial observer sees the same events:

$$
\forall m, k \in \mathrm{IOb} \quad E v_{m}=E v_{k} .
$$

One can prove from $\mathrm{AxPh}_{0}$ and AxEOF that if $m$ is an inertial observer and $e \in E v_{m}$, then there is a unique coordinate point $\vec{p} \in \mathrm{Q}^{d}$ such that $e=e v_{m}(\vec{p})$. We will denote this unique coordinate point $\vec{p} \in \mathrm{Q}^{d}$ by $\operatorname{Crd}_{m}(e)$.

Convention 4.1. Whenever we write " $\mathrm{Cr} d_{m}(e)$ ", we mean that there is a unique $\vec{q} \in C d_{m}$ such that $e v_{m}(\vec{q})=e$, and $C r d_{m}(e)$ denotes this unique $\vec{q}$. That is, if we talk about the value $\operatorname{Crd}_{m}(e)$, we postulate that it exists and is unique (by the present convention).

We say that events $e_{1}$ and $e_{2}$ are simultaneous for observer $m$, in symbols $e_{1} \sim_{m} e_{2}$, iff $e_{1}$ and $e_{2}$ have the same time-coordinate in $m$ 's coordinate-domain, i.e. if $\operatorname{Crd}_{m}\left(e_{1}\right)_{t}=\operatorname{Crd}_{m}\left(e_{2}\right)_{t}$. To talk about time differences measured by observers, we use time ${ }_{m}\left(e_{1}, e_{2}\right)$ as an abbreviation for $\left|\operatorname{Crd}_{m}\left(e_{1}\right)_{t}-\operatorname{Crd}_{m}\left(e_{2}\right)_{t}\right|$ and we call it the elapsed time between events $e_{1}$ and $e_{2}$ measured by observer $m$. We note that, if $m \in e_{1} \cap e_{2}$, then time ${ }_{m}\left(e_{1}, e_{2}\right)$ is called the proper time measured by $m$ between $e_{1}$ and $e_{2}$, and $e_{1} \sim_{m} e_{2}$ iff time ${ }_{m}\left(e_{1}, e_{2}\right)=0$. We use $\operatorname{dist}_{m}\left(e_{1}, e_{2}\right)$ as an abbreviation for $\left|\operatorname{Crd}_{m}\left(e_{1}\right)_{s}-\operatorname{Crd}_{m}\left(e_{2}\right)_{s}\right|$ and we call it the spatial distance of events $e_{1}$ and $e_{2}$ according to an observer $m$. We note that when we write $\operatorname{dist}_{m}\left(e_{1}, e_{2}\right)$ or time $m\left(e_{1}, e_{2}\right)$, we assume that $e_{1}$ and $e_{2}$ have unique coordinates by Convention 4.1.

AxSimDist: If events $e_{1}$ and $e_{2}$ are simultaneous for both inertial observers $m$ and $k$, then $m$ and $k$ agree on the spatial distance between $e_{1}$ and $e_{2}$ :

$$
\begin{aligned}
\forall m, k \in \mathrm{IOb} \forall e_{1}, e_{2} \in E v_{m} \quad & \left(e_{1} \sim_{m} e_{2} \wedge e_{1} \sim_{k} e_{2} \Longrightarrow\right. \\
& \left.\operatorname{dist}_{m}\left(e_{1}, e_{2}\right)=\operatorname{dist}_{k}\left(e_{1}, e_{2}\right)\right) .
\end{aligned}
$$

Let us collect these axioms in an axiom system called $\mathrm{SpecRe}_{d}$.

$$
\text { SpecRel }_{d}:=\left\{\text { AxFrame, AxEOF, AxSelf }{ }^{-}, \text {AxPh }_{0}, \text { AxEv, AxSimDist }\right\} .
$$

Now for each natural number $d \geq 2$, we have a FOL theory of SR. Usually we omit the dimension parameter $d$. From the few axioms introduced so far, we can deduce the most frequently quoted predictions, called paradigmatic effects, of SR: (i) "moving clocks slow down" (ii) "moving meter-rods shrink" (iii) "moving pairs of clocks get out of synchronism". For more detail, see for example, 1], [2, or [3]. Here we concentrate on the behavior of clocks and indicate a connection with Minkowski geometry. 
Theorem 4.2. Assume SpecRel $_{d}, d \geq 3$. Then

$$
\text { time }_{m}\left(e_{1}, e_{2}\right)^{2}-\operatorname{dist}_{m}\left(e_{1}, e_{2}\right)^{2}=\operatorname{time}_{k}\left(e_{1}, e_{2}\right)^{2}-\operatorname{dist}_{k}\left(e_{1}, e_{2}\right)^{2}
$$

for any $m, k \in \mathrm{IOb}$ and $e_{1}, e_{2} \in E v_{m}$.

The above theorem is the starting point for building Minkowski geometry, which is the "geometrization" of SR. It also indicates that time and space are intertwined in SR. Here we only concentrate on its corollary usually stated as "moving clocks slow down". Theorem 4.2 shows that SpecRel is a good axiom system for SR if we restrict our interest to inertial motion.

Corollary 4.3. (moving clocks slow down) Assume SpecRel $d, d \geq 3$. Let $m, k \in \mathrm{IOb}, e_{1}, e_{2} \in E v_{k}$, and assume $k \in e_{1} \cap e_{2}, \operatorname{dist}_{m}\left(e_{1}, e_{2}\right) \neq 0$. Then

$$
\text { time }_{m}\left(e_{1}, e_{2}\right)>\operatorname{time}_{k}\left(e_{1}, e_{2}\right) .
$$

In the above corollary, a "moving clock" is represented by observer $k$, that he is moving relative to $m$ is expressed by $\operatorname{dist}_{m}\left(e_{1}, e_{2}\right) \neq 0$, $k \in e_{1} \cap e_{2}$, and that $k$ 's time is slowing down relative to $m$ 's is expressed by time $e_{m}\left(e_{1}, e_{2}\right)>$ time $_{k}\left(e_{1}, e_{2}\right)$. This "clock slowing down" effect is only relative, i.e., "clocks moving relative to $m$ slow down relative to $m$ ". But this relative effect leads to a new kind of gravitation-oriented "absolute slowing time down" effect, as our next theorem as well as the whole of section [5] will show.

To extend SpecRel, we now formulate axioms about non-inertial observers. The non-inertial observers are called accelerated observers. Note that AxSelf ${ }^{-}$is the only axiom introduced so far that talks about non-inertial observers, too. We assume the following very natural axiom for all observers.

$\mathrm{AxEv}^{+}$: Whenever an observer participates in an event, he also sees this event:

$$
\forall m \in \mathrm{Ob} \forall e \in E v \quad\left(m \in e \Longrightarrow e \in E v_{m}\right) .
$$

The set of positive elements of $\mathrm{Q}$ is denoted by $\mathrm{Q}^{+}:=\{x \in \mathrm{Q}: x>0\}$. The interval between $x, y \in \mathrm{Q}$ is defined as $(x, y):=\{z \in \mathrm{Q}: x<z<$ $y$ \}. Let $H \subseteq \mathrm{Q}$. We say that $H$ is connected iff $\forall x, y \in H(x, y) \subseteq H$, and we say that $H$ is open iff $\forall x \in H \quad \exists \varepsilon \in \mathrm{Q}^{+}(x-\varepsilon, x+\varepsilon) \subseteq H$.

We assume the following technical axiom:

$\mathrm{AxSelf}^{+}$: The set of time-instances in which an observer is present in its own world-view is connected and open:

$$
\forall m \in \mathrm{Ob}\left\{p_{t}: m \in e v_{m}(\vec{p})\right\} \quad \text { is connected and open. }
$$

To connect the coordinate-domains of the accelerated and the inertial observers, we are going to formulate the statement that at each moment of his life, each accelerated observer sees the nearby world for a short while as an inertial observer does. To formalize this, first we introduce 
the relation of being a co-moving observer. To do so, we define the (coordinate) neighborhood of event $e$ with radius $r \in \mathrm{Q}^{+}$according to observer $k$ as:

$$
B_{k}^{r}(e):=\left\{\vec{p} \in C d_{k}: \exists \vec{q} \in C d_{k} \quad e v_{k}(\vec{q})=e \wedge|\vec{p}-\vec{q}|<r\right\} .
$$

We note that $B_{k}^{r}(e)=\emptyset$ if $e \notin E v_{k}$ by this definition. Observer $m$ is a co-moving observer of observer $k$ at event $e$, in symbols $m \succ_{e} k$, iff the following holds:

$\forall \varepsilon \in \mathrm{Q}^{+} \exists \delta \in \mathrm{Q}^{+} \forall \vec{p} \in B_{k}^{\delta}(e) \quad\left|\vec{p}-\operatorname{Crd}_{m}\left(\operatorname{ev}_{k}(\vec{p})\right)\right| \leq \varepsilon\left|\vec{p}-\operatorname{Crd}_{k}(e)\right|$.

Note that $\operatorname{Crd}_{m}(e)=\operatorname{Crd}_{k}(e)$ and thus also $e \in E v_{m}$ if $m \succ_{e} k$ and $e \in E v_{k}$. Note also that $m \succ_{e} k$ for every observer $m$ if $e \notin E v_{k}$, by definition. Behind the definition of the co-moving observers is the following intuitive image: as we zoom into smaller and smaller neighborhoods of the coordinate point of the given event, the coordinatedomains of the two observers are more and more similar. This intuitive picture is symmetric while the co-moving relation $\succ_{e}$ is not. Thus we introduce a symmetric version. We say that observers $m$ and $k$ are strong co-moving observers at event $e$, in symbols $m *_{e} k$, iff both $m \succ_{e} k$ and $k \succ_{e} m$ hold. The following axiom gives the promised connection between the coordinate-domains of the inertial and the accelerated observers:

$\mathrm{AxAcc}^{+}$: At any event in which an observer sees himself, there is a strong co-moving inertial observer.

$$
\forall k \in \mathrm{Ob} \forall e \in E v \quad\left(k \in e \Longrightarrow \exists m \in \mathrm{IOb} m \varkappa_{e} k\right) .
$$

The axioms introduced so far are not strong enough to prove properties of accelerated clocks like the Twin Paradox, cf. Theorems 3.5 and 3.7 and Corollary 3.6 in 44. The additional property we need is that every bounded non-empty subset of the quantity part has a supremum. This is a second-order logic property (because it concerns all subsets) which we cannot use in a FOL axiom system. Instead, we will use a kind of "induction" axiom schema. It will state that every non-empty, bounded subset of the quantity part which can be defined by a FOL-formula using possibly the extra part of the model, e.g., using the world-view relation, has a supremum. To formulate this FOL induction axiom schema, we need some more definitions.

If $\varphi$ is a formula and $x$ is a variable, then we say that $x$ is a free variable of $\varphi$ iff $x$ does not occur under the scope of either $\exists x$ or $\forall x$. Sometimes we introduce a formula $\varphi$ as $\varphi(\vec{x})$, this means that all the free variables of $\varphi$ lie in $\vec{x}$.

If $\varphi(x, y)$ is a formula and $\mathfrak{M}=\langle U ; \ldots\rangle$ is a model, then whether $\varphi$ is true or false in $\mathfrak{M}$ depends on how we associate elements of $U$ to the free variables $x, y$. When we associate $a, b \in U$ to $x, y$, respectively, then $\varphi(a, b)$ denotes this truth-value, thus $\varphi(a, b)$ is either true or false in $\mathfrak{M}$. For example, if $\varphi$ is $x \leq y$, then $\varphi(0,1)$ is true while $\varphi(1,0)$ is 
false in any ordered field. A formula $\varphi$ is said to be true in $\mathfrak{M}$ if $\varphi$ is true in $\mathfrak{M}$ no matter how we associate elements to the free variables. We say that a subset $H$ of $\mathrm{Q}$ is (parametrically) definable by $\varphi(y, \vec{x})$ iff there is $\vec{a} \in U^{n}$ such that $H=\{b \in \mathrm{Q}: \varphi(b, \vec{a})$ is true in $\mathfrak{M}\}$. We say that a subset of $\mathrm{Q}$ is definable iff it is definable by a FOL-formula.

Let $\phi(x, \vec{y})$ be a FOL-formula of our language.

AxSup $_{\phi}$ : Every subset of $\mathrm{Q}$ definable by $\phi(x, \vec{y})$ has a supremum if it is non-empty and bounded.

A FOL formula expressing $\operatorname{AxSup}_{\phi}$ can be found in [44. Our axiom scheme IND below says that every non-empty bounded subset of $\mathrm{Q}$ that is definable in our language has a supremum:

$$
\text { IND }:=\left\{\operatorname{AxSup}_{\varphi}: \varphi \text { is a FOL-formula of our language }\right\} .
$$

Note that IND is true in any model whose quantity part is the field of real numbers. For more detail about IND cf. 44.

Let us call the collection of the axioms introduced so far AccRel $_{d}$ :

$$
\text { AccRel }_{d}:=\text { SpecRel }_{d} \cup\left\{\mathrm{AxEv}^{+}, \mathrm{AxSelf}^{+}, \mathrm{AxAcc}^{+}\right\} \cup \mathrm{IND} .
$$

The so-called Twin Paradox is provable in AccRel, cf. 44, 61. We now formulate the Twin Paradox with our logical notation.

The set of events encountered by $m \in \mathrm{Ob}$ between $e_{1}, e_{2} \in E v$ is denoted as

$$
\begin{aligned}
E v_{m}\left(e_{1}, e_{2}\right):= & \left\{e \in E v_{m}: m \in e \wedge\right. \\
& \left.\operatorname{Crd}_{m}\left(e_{1}\right)_{t}<\operatorname{Crd}_{m}(e)_{t}<\operatorname{Crd}_{m}\left(e_{2}\right)_{t}\right\} .
\end{aligned}
$$

Now we can formulate the Twin Paradox in our FOL setting.

TwP: Every inertial observer $m$ measures more time than or equal time as any other observer $k$ between any two meeting events $e_{1}$ and $e_{2}$; and they measure the same time iff they have encountered the same events between $e_{1}$ and $e_{2}$ :

$$
\begin{aligned}
\forall e_{1}, e_{2} \in E v \forall m \in \operatorname{IOb} \forall k \in \mathrm{Ob} & \left(k, m \in e_{1} \cap e_{2} \Longrightarrow\right. \\
\left(\operatorname{time}_{m}\left(e_{1}, e_{2}\right)=\operatorname{time}_{k}\left(e_{1}, e_{2}\right)\right. & \left.\Longleftrightarrow E v_{m}\left(e_{1}, e_{2}\right)=E v_{k}\left(e_{1}, e_{2}\right)\right) \\
& \left.\wedge \operatorname{time}_{m}\left(e_{1}, e_{2}\right) \geq \operatorname{time}_{k}\left(e_{1}, e_{2}\right)\right) .
\end{aligned}
$$

The following theorem states that the Twin Paradox is provable in AccRel $_{d}$ if $d \geq 3$.

Theorem 4.4. AccRel $_{d}=\mathrm{TwP}$, if $d \geq 3$.

For the proof of this theorem, cf. [44, 61].

We note that there are non-trivial models of AccRel. E.g., the construction in Misner-Thorne-Wheeler [46, §6, especially pp. 172-173 and $§ 13.6$ on pp. 327-332] can be used for constructing models for AccRel. 


\section{One STEP tOWARD GR (EFFECT OF GRAVITATION ON ClOCKS)}

We would like to investigate the effect of gravitation on clocks in our FOL setting. As a first step we prove theorems about the Gravitational Time Dilation that roughly says that "gravitation makes time flow slower", that is, the clocks in the bottom of a tower run slower than the clocks in the top of the tower. We will use Einstein's equivalence principle to treat gravitation in AccRel. This principle says that a uniformly accelerated frame of reference is indistinguishable from a rest frame in a uniform gravitational field, cf. e.g., d'Inverno [12, §9.4]. So instead of gravitation we will talk about acceleration and instead of towers we will talk about spaceships. This way the Gravitational Time Dilation will become the following statement: "the time in the aft of an accelerated spaceship flows slower than in the front of the spaceship". We begin to formulate this statement in our FOL language.

To talk about spaceships, we will need a concept of distance between events and observers. We have the following two natural candidates for this:

- Event $e$ is at radar-distance $\lambda \in \mathrm{Q}^{+}$from observer $k$ iff there are events $e_{1}$ and $e_{2}$ and photons $p h_{1}$ and $p h_{2}$ such that $k \in$ $e_{1} \cap e_{2}, p h_{1} \in e \cap e_{1}, p h_{2} \in e \cap e_{2}$ and $\operatorname{time}_{k}\left(e_{1}, e_{2}\right)=2 \lambda$. Event $e$ is at radar-distance 0 from observer $k$ iff $k \in e$. See $(a)$ of Figure 2 .

- Event $e$ is at Minkowski-distance $\lambda \in \mathrm{Q}$ from observer $k$ iff there is an event $e^{\prime}$ such that $k \in e^{\prime}, e \sim_{m} e^{\prime}$ and $\operatorname{dist}_{m}\left(e, e^{\prime}\right)=\lambda$ for every inertial co-moving observer $m$ of $k$ at $e^{\prime}$. See (b) of Figure 2.

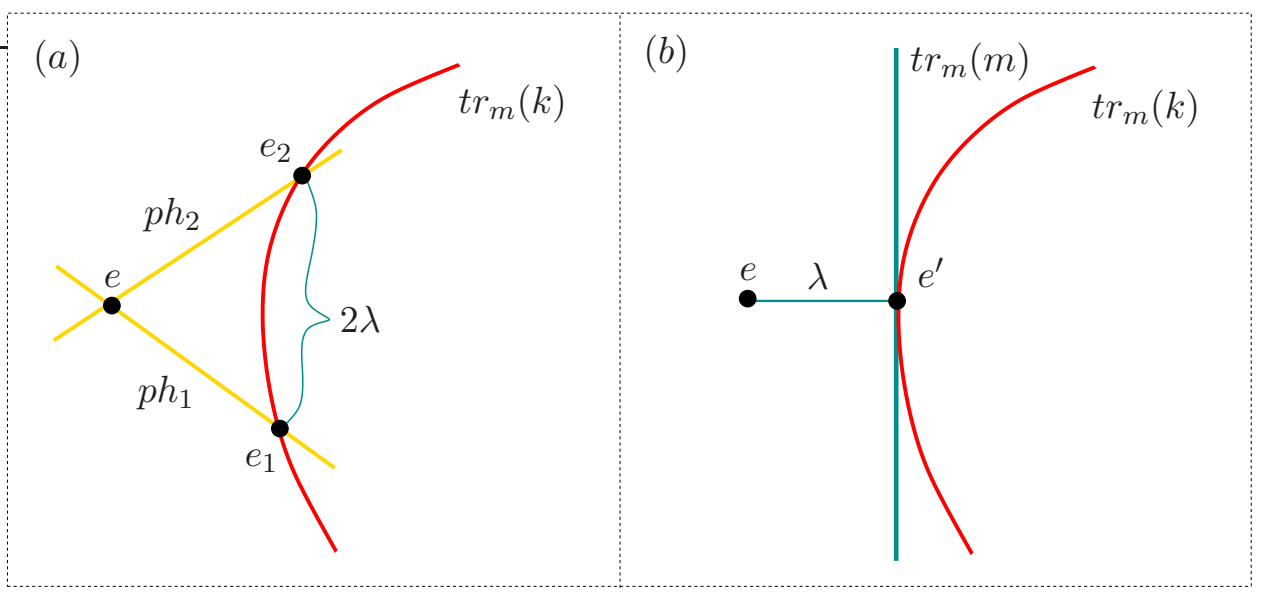

Figure 2. (a) for the radar-distance and $(b)$ for the Minkowski-distance. 
We say that observer $k$ thinks that body $b$ is at constant radar (Minkowski) distance from him iff the radar-distance (Minkowski-distance) of every event which $b$ participates in is the same.

The life-line ${ }^{2}$ (or trace) of body $b$ according to observer $m$ is defined as the set of coordinate points where $b$ was observed by $m$ :

$$
\operatorname{tr}_{m}(b):=\left\{\vec{p} \in \mathrm{Q}^{d}: \mathrm{W}(m, b, \vec{p})\right\} .
$$

Note that $\operatorname{tr}_{m}(b)=\left\{\vec{p} \in \mathrm{Q}^{d}: b \in e v_{m}(\vec{p})\right\}$. For stating that the spaceship does not change its direction we introduce the following concept. We say that observers $k$ and $b$ are coplanar iff $t r_{m}(k) \cup t r_{m}(b)$ is a subset of a plane containing a line parallel with the time-axis, in the coordinate system of an inertial observer $m$.

We now introduce two concepts for spaceships. Observers $b, k$ and $c$ form a radar-spaceship, in symbols $\rangle|b, k, c\rangle_{\text {rad }}$, iff $b, k$ and $c$ are coplanar and $k$ thinks that $b$ and $c$ are at constant radar-distances from him. The definition of the Minkowski-spaceship, in symbols $>b, k, c\rangle_{\mu}$, is analogous.

We say that event $e_{1}$ (causally) precedes event $e_{2}$ according to observer $k$ iff $\operatorname{Crd}_{m}\left(e_{1}\right)_{t} \leq \operatorname{Crd}_{m}\left(e_{2}\right)_{t}$ for all inertial co-moving observers $m$ of $k$. In this case, we also say that $e_{2}$ succeeds $e_{1}$ according to $k$.

We need some concept for deciding which events happened at the same time according to an accelerated observer. The following three natural concepts offer themselves:

- Events $e$ and $e^{\prime}$ are radar-simultaneous for observer $k$, in symbols $e \sim_{k}^{\text {rad }} e^{\prime}$, iff $k \in e$ and there are events $e_{1}$ and $e_{2}$ and photons $p h_{1}$ and $p h_{2}$ such that $k \in e_{1} \cap e_{2}, p h_{1} \in e \cap e_{1}$, $p h_{2} \in e \cap e_{2}$ and $\operatorname{time}_{k}\left(e_{1}, e\right)=\operatorname{time}_{k}\left(e, e_{2}\right)$ or there is an event $e_{3}$ such that $e \sim_{k}^{r a d} e_{3}$ and $e_{3} \sim_{k}^{r a d} e^{\prime}$. See $(a)$ of Figure 3 ,

- Events $e_{1}$ and $e_{2}$ are photon-simultaneous for observer $k$, in symbols $e_{1} \sim_{k}^{p h} e_{2}$, iff there is an event $e$ and photons $p h_{1}$ and $p h_{2}$ such that $k \in e, p h_{1} \in e \cap e_{1}, p h_{2} \in e \cap e_{2}$ and $e_{1}$ and $e_{2}$ precedes $e$ according to $k$. See $(b)$ of Figure 3 .

- Events $e_{1}$ and $e_{2}$ are Minkowski-simultaneous for observer $k$, in symbols $e_{1} \sim_{k}^{\mu} e_{2}$, iff there is an event $e$ such that $k \in e$ and $e_{1}$ and $e_{2}$ are simultaneous for any inertial co-moving observer of $k$ at $e$. See $(c)$ of Figure 3

We note that, for inertial observers, the concepts of radar-simultaneity and Minkowski-simultaneity coincide with the concept of simultaneity introduced on page 10 .

We will distinguish the front and the aft of the spaceship by the direction of the acceleration. Thus we need a concept for direction. We say that the directions of $\vec{p} \in \mathrm{Q}^{d}$ and $\vec{q} \in \mathrm{Q}^{d}$ are the same,

\footnotetext{
${ }^{2}$ Life-line is called world-line in some of the literature.
} 


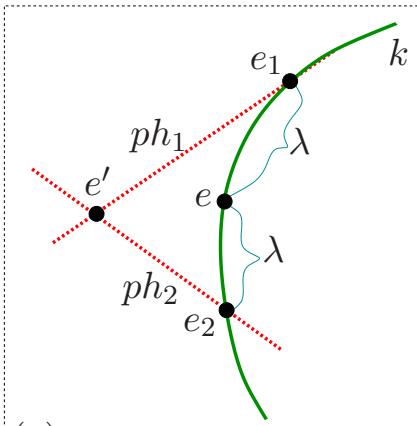

(a)
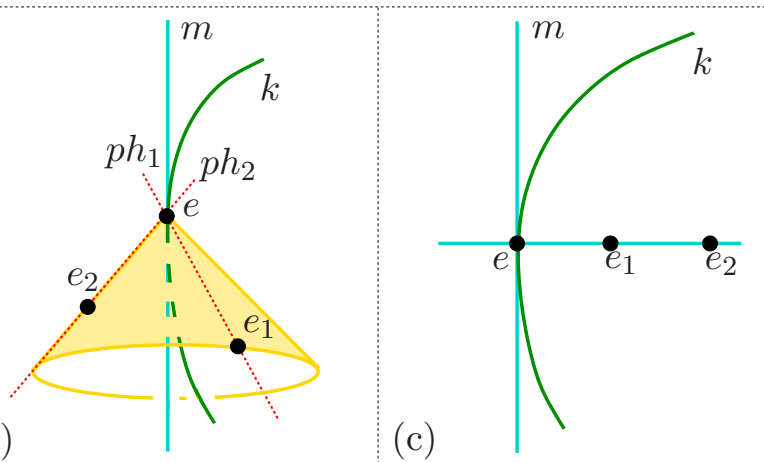

FiguRE 3. (a) is for $e \sim_{k}^{r a d} e^{\prime},(b)$ is for $e_{1} \sim_{k}^{p h} e_{2}$ and (c) is for $e_{1} \sim_{k}^{\mu} e_{2}$.

in symbols $\vec{p} \uparrow \uparrow \vec{q}$, iff there is a $\lambda \in \mathrm{Q}^{+}$such that $\lambda \vec{p}_{s}=\vec{q}_{s}$, see $(a)$ of Figure 4.

Now let us turn our attention towards the definition of acceleration in our FOL setting.

We define the life-curve of observer $k$ according to observer $m$ as the life-line of $k$ according to $m$ parameterized by the time measured by $k$, formally:

$$
\begin{aligned}
T r_{m}^{k} & :=\left\{\langle t, \vec{p}\rangle \in \mathrm{Q} \times C d_{m}:\right. \\
& \left.\exists \vec{q} \in t r_{k}(k) \quad q_{t}=t \wedge e v_{m}(\vec{p})=e v_{k}(\vec{q})\right\} .
\end{aligned}
$$

The domain of a binary relation $R$ is defined as Dom $R:=\{x: \exists y\langle x, y\rangle \in$ $R\}$.

Both the life-curves of observers (according to any inertial observer) and the derivative $f^{\prime}$ of a given function $f$ are first-order logic definable concepts, cf. 44]. Thus the following definitions are also FOL ones: The relative-velocity $\vec{v}_{m}^{k}$ of observer $k$ according to observer $m$ at instant $t \in \mathrm{Q}$ is the derivative of the life-curve of $k$ according to $m$ at $t$, i.e., $\vec{v}_{m}^{k}(t)=\left(\operatorname{Tr}_{m}^{k}\right)^{\prime}(t)$ if $t \in \operatorname{Dom} \operatorname{Tr}_{m}^{k}$ and undefined otherwise. The relative-acceleration $\vec{a}_{m}^{k}$ of observer $k$ according to observer $m$ at instant $t \in \mathrm{Q}$ is the derivative of the relative-velocity at $t$ if it is differentiable at $t$ and undefined otherwise.

Events $e_{1}$ and $e_{2}$ are called spacelike separated, in symbols $e_{1} \equiv{ }_{s}$ $e_{2}$, iff $\operatorname{Crd}_{m}\left(e_{1}\right)$ and $\operatorname{Crd}_{m}\left(e_{2}\right)$ can be connected by a line of slope less than 1 for every inertial observer $m$, i.e., iff $\left|\left(\operatorname{Crd}_{m}\left(e_{1}\right)-\operatorname{Crd}_{m}\left(e_{2}\right)\right)_{s}\right|>$ $\left|\left(\operatorname{Crd}_{m}\left(e_{1}\right)-\operatorname{Crd}_{m}\left(e_{2}\right)\right)_{t}\right|$ for every inertial observer $m$. We say that the direction of the spaceship $\rangle|b, k, c\rangle$ agrees with that of the acceleration of $k$ iff the following holds:

$$
\begin{aligned}
& \forall m \in \text { IOb } \forall t \in \text { Dom } \vec{a}_{m}^{k} \forall e_{1}, e_{2} \in E v \quad\left(c \in e_{1} \wedge\right. \\
& \left.b \in e_{2} \wedge e_{1} \equiv_{s} e_{2} \Longrightarrow \vec{a}_{m}^{k}(t) \uparrow \uparrow\left(\operatorname{Crd}_{k}\left(e_{1}\right)-\operatorname{Crd}_{k}\left(e_{2}\right)\right)\right) .
\end{aligned}
$$




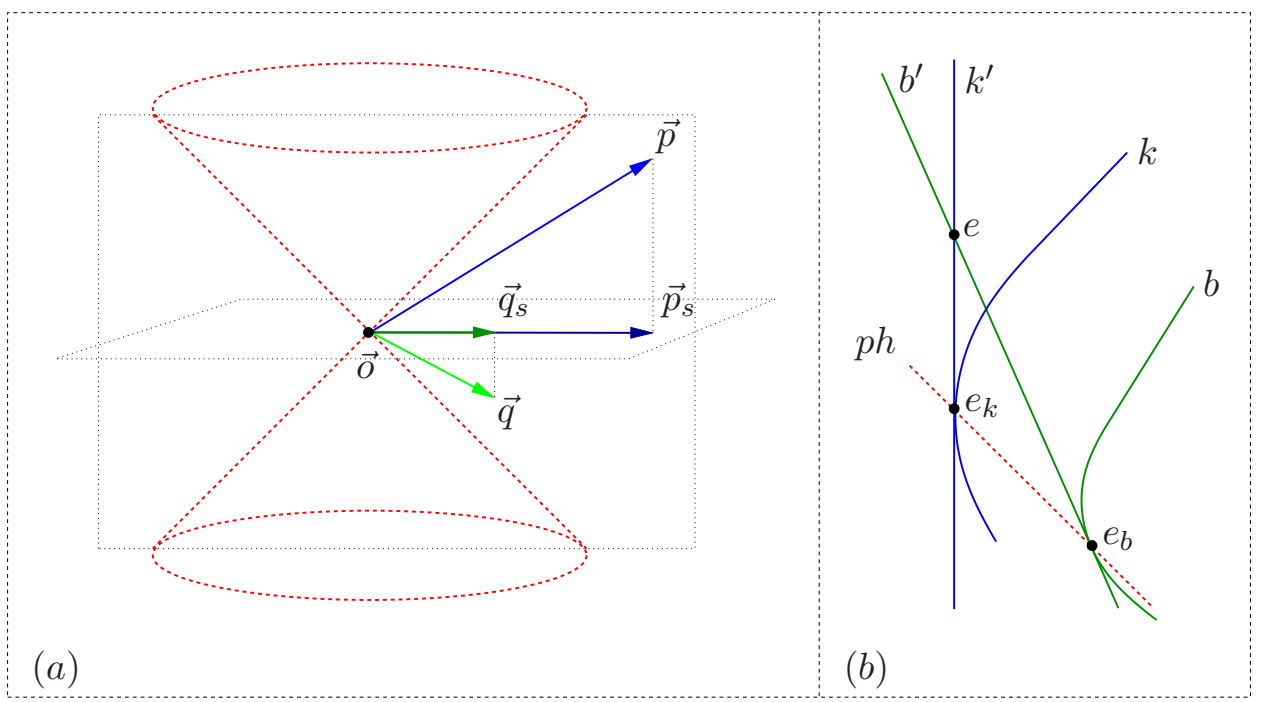

FiguRE 4. (a) is for illustrating $\vec{p} \uparrow \uparrow \vec{q}$ and $(b)$ is for illustrating observer $b$ approaching to observer $k$, as seen by $k$ with photons.

The (signed) Minkowski-length of $\vec{p} \in \mathrm{Q}^{d}$ is

$$
\mu(p):=\left\{\begin{aligned}
\sqrt{\left.\left|p_{t}^{2}-\right| \vec{p}_{s}\right|^{2} \mid} & \text { if } p_{t}^{2}-\left|\vec{p}_{s}\right|^{2} \geq 0, \\
-\sqrt{\left.\left|p_{t}^{2}-\right| \vec{p}_{s}\right|^{2} \mid} & \text { otherwise }
\end{aligned}\right.
$$

and the Minkowski-distance between $\vec{p}$ and $\vec{q}$ is $\mu(\vec{p}, \vec{q}):=\mu(\vec{p}-\vec{q})$. A motivation for the "otherwise" part of the definition of $\mu(\vec{p})$ is the following. $\mu(\vec{p})$ codes two kinds of information, (i) the length of $\vec{p}$ and (ii) whether $\vec{p}$ is timelike (i.e. $\left|p_{t}\right|>\left|\vec{p}_{s}\right|$ ) or spacelike. Since the length is always non-negative, we can use the sign of $\mu(\vec{p})$ to code (ii).

The acceleration of an observer $k$ at instant $t \in \mathrm{Q}$ is defined as the Minkowski-length of the relative-acceleration seen by any inertial observer $m$ at $t$, that is:

$$
a_{k}(t):=\mu\left(\vec{a}_{m}^{k}(t)\right) .
$$

The acceleration is a well defined concept since it is independent of the choice of the inertial observer $m$. We say that observer $k$ is positively

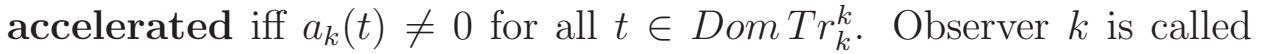
uniformly accelerated iff there is an $a \in \mathrm{Q}^{+}$such that $a_{k}(t)=a$ for all $t \in \operatorname{Dom} \operatorname{Tr}_{k}^{k}$.

We say that the clock of $b$ runs slower than the clock of $c$ as seen by $k$ with radar (photons; Minkowski-simultaneity) iff time $_{b}\left(e_{b}, e_{b}^{\prime}\right)<$ time $_{c}\left(e_{c}, e_{c}^{\prime}\right)$ for all events $e_{b}, e_{b}^{\prime}, e_{c}, e_{c}^{\prime}$ for which $b \in e_{b} \cap e_{b}^{\prime}$, $c \in e_{c} \cap e_{c}^{\prime}$ and $e_{b} \sim_{k}^{r a d} e_{c}, e_{b}^{\prime} \sim_{k}^{r a d} e_{c}^{\prime} .\left(e_{b} \sim_{k}^{p h} e_{c}, e_{b}^{\prime} \sim_{k}^{p h} e_{c}^{\prime} ; e_{b} \sim_{k}^{\mu} e_{c}\right.$, $\left.e_{b}^{\prime} \sim_{k}^{\mu} e_{c}^{\prime}\right)$. 
Now we can state our first theorem about the clock-slowing effect of gravitation:

Theorem 5.1. Assume $\mathrm{AccRel}_{d}$ and $d \geq 3$. Let $>|b, k, c\rangle_{\text {rad }}$ be a radarspaceship such that:

(1) $k$ is positively accelerated,

(2) the direction of the spaceship agrees with that of the acceleration of $k$.

(i) Then the clock of $b$ runs slower than the clock of $c$ as seen by $k$ with radar and $(i i)$ the clock of $b$ runs slower than the clock of $c$ as seen by each of $k, b$ and $c$ with photons.

To state a similar theorem in Minkowski-spaceships, we need the following concept. We say that observer $b$ is not too far behind positively accelerated observer $k$ iff the following holds:

$$
\begin{array}{r}
\forall m \in \text { IOb } \forall t \in D o m \operatorname{Tr}_{m}^{k} \forall \vec{p}, \vec{q} \in C d_{m} \quad\left(\vec{p} \in t r_{m}(k) \wedge\right. \\
\vec{q} \in t r_{m}(b) \wedge e v_{m}(\vec{p}) \sim_{k}^{\mu} e v_{m}(\vec{q}) \wedge \vec{a}_{m}^{k}(t) \uparrow \uparrow(\vec{p}-\vec{q}) \Longrightarrow \\
\left.\forall \tau \in \operatorname{Dom} \vec{a}_{m}^{k} \quad \mu(\vec{p}-\vec{q})<1 / a_{k}(\tau)\right) .
\end{array}
$$

Now we can state our second theorem about the clock-slowing effect of gravitation:

Theorem 5.2. Assume AccRel $_{d}$ and $d \geq 3$. Let $>|b, k, c\rangle_{\mu}$ be a Minkowskispaceship such that:

(1) $k$ is positively accelerated,

(2) the direction of the spaceship agrees with that of the acceleration of $k$,

(3) $b$ is not too far behind $k$.

Then $(i)$ the clock of $b$ runs slower than the clock of $c$ as seen by $k$ with Minkowski-simultaneity or with photons and $(i i)$ the clock of $b$ runs slower than the clock of $c$ as seen by each of $k, b$ and $c$ with photons.

In the following theorem we will see that the flow of time as seen by photons is strongly connected with the following two concepts. We say that observer $b$ is approaching to (moving away from) observer $k$ as seen by $k$ with photons iff for all events $e_{k}$ and $e_{b}$, if $b \in e_{b}, k \in e_{k}$ and $e_{k} \sim_{k}^{p h} e_{b}$, then there is an event $e$ such that $k^{\prime}, b^{\prime} \in e$ for all inertial comoving observers $k^{\prime}$ and $b^{\prime}$ of $k$ and $b$ at events $e_{k}$ and $e_{b}$, respectively, and $e_{b}$ precedes (succeeds) $e_{k}$ according to $k$, cf. (b) of Figure 4 .

We say that the life-curve of observer $k$ is continuously differentiable if the curve $\operatorname{Tr}_{m}^{k}$ is such for all inertial observers $m$.

Theorem 5.3. Assume AccRel $_{d}$ and $d \geq 3$. Let $b, k \in \mathrm{Ob}$ such that $b$ and $k$ are coplanar and the life-curve of $k$ is continuously differentiable.

(1) If $b$ is approaching to $k$ as seen by $k$ with photons, then the clock of $k$ runs slower than the clock of $b$ as seen by $k$ with photons. 
(2) If $b$ is moving away from $k$ as seen by $k$ with photons, then the clock of $b$ runs slower than the clock of $k$ as seen by $k$ with photons.

None of the axioms introduced so far require the existence of accelerated (non-inertial) observers. Our following axiom scheme says that every definable timelike curve is the life-line of an observer. Since from $\mathrm{AxSelf}{ }^{-}, \mathrm{AxPh}_{0}$ and $\mathrm{AxEv}$ it follows that the life-lines of inertial observers are straight lines, cf. e.g., [1, 39, 40], this will ensure the existence of many non-inertial observers.

A differentiable function $\gamma$ is called timelike curve iff the slope of $\gamma^{\prime}(t)$ is less than 1 (i.e., $\left.\left.\mid \gamma^{\prime}(t)\right)_{s}|<| \gamma^{\prime}(t)\right)_{t} \mid$ ) for all $t \in D o m \gamma$ and Dom $\gamma$ is an open and connected subset of Q. It is clear that this is a first-order logic definable concept since every fragment of it is such. We say that a function $f$ is (parametrically) definable by $\psi(x, \vec{y}, \vec{z})$ iff there is $\vec{a} \in U^{n}$ such that $f(b)=\vec{p} \Longleftrightarrow \psi(b, \vec{p}, \vec{z})$ true in $\mathfrak{M}$.

Let $\psi$ be a FOL-formula of our language.

$\mathrm{A} \times \exists \mathrm{Ob}_{\psi}$ : If a function parametrically definable by $\psi$ is a timelike curve, then there is an observer whose life-line is the range of this function.

$\mathrm{A} \times \exists \mathrm{Ob}:=\left\{\mathrm{A} \times \exists \mathrm{Ob}_{\psi}: \psi\right.$ is a FOL-formula of our language $\}$.

The following three theorems say that the clocks can run arbitrarily slow or fast, as seen with the three different methods.

Theorem 5.4. Assume $\operatorname{AccRel}_{d}, \mathrm{Ax} \exists \mathrm{Ob}$ and $d \geq 3$. Let $k \in \mathrm{Ob}$ be positively accelerated such that $\operatorname{Dom~}_{\mathrm{Tr}_{k}^{k}}=\mathrm{Q}$ and let $e, e^{\prime} \in E v$ such that $e \neq e^{\prime}$ and $m \in e \cap e^{\prime}$. Then for all $\lambda \in \mathrm{Q}^{+}$, there are an observer $b$ and events $e_{b}, e_{b}^{\prime} \in E v$ such that $b \in e_{b} \cap e_{b}^{\prime}, e \sim_{m}^{r a d} e_{b}, e^{\prime} \sim_{m}^{r a d} e_{b}^{\prime}$ and time $_{b}\left(e_{b}, e_{b}^{\prime}\right)=\lambda$ time $_{m}\left(e, e^{\prime}\right)$.

Theorem 5.5. Assume AccRel $_{d}$ and $d \geq 3$. Let $k \in \mathrm{Ob}$ be uniformly accelerated and let $e, e^{\prime} \in E v$ such that $e \neq e^{\prime}$ and $m \in e \cap e^{\prime}$. Then for all $\lambda \in \mathrm{Q}^{+}$, there are an observer $b$ and events $e_{b}, e_{b}^{\prime} \in E v$ such that $b \in e_{b} \cap e_{b}^{\prime}, e \sim_{m}^{\mu} e_{b}, e^{\prime} \sim_{m}^{\mu} e_{b}^{\prime}$ and $\operatorname{time}_{b}\left(e_{b}, e_{b}^{\prime}\right)=\lambda \operatorname{time}_{m}\left(e, e^{\prime}\right)$.

Theorem 5.6. Assume $\mathrm{AccRe}_{d}, \mathrm{Ax} \exists \mathrm{Ob}$ and $d \geq 3$. Let $k \in \mathrm{Ob}$ be positively accelerated and $e, e^{\prime} \in E v$ such that $e \neq e^{\prime}$ and $m \in e \cap e^{\prime}$. Then for all $\lambda \in \mathrm{Q}^{+}$, there are an observer $b$ and events $e_{b}, e_{b}^{\prime} \in E v$ such that $b \in e_{b} \cap e_{b}^{\prime}, e \sim_{m}^{p h} e_{b}, e^{\prime} \sim_{m}^{p h} e_{b}^{\prime}$ and $\operatorname{time}_{b}\left(e_{b}, e_{b}^{\prime}\right)=\lambda$ time $_{m}\left(e, e^{\prime}\right)$.

We have seen that gravitation (acceleration) makes "time flow slowly". However, we left open the question what role the "strength" and the "direction" of the gravitation play in this effect. The following theorem shows that two observers, say $m$ and $k$, can feel the same gravitation while the clock of $k$ runs slower than the clock of $m$. Thus it is not the "strength" of the gravitation that makes "time flow more slowly". 
Theorem 5.7. Assume $\mathrm{AccRel}_{d}, \mathrm{~A} \times \exists \mathrm{Ob}$ and $d \geq 3$. There are uniformly accelerated observers $m$ and $k$ such that $a_{k}(t)=a_{m}(t)$ for all $t \in \mathrm{Q}$, but the clock of $k$ runs slower than the clock of $m$ as seen by both $m$ and $k$ with photons (or with radar or with Minkowski simultaneity).

Now let us see what we can say about the role of the "direction" of gravitation. Being "more down in a gravitational well" becomes being "behind" if we translate it from the language of gravitation into the language of acceleration. This can be formulated by our notation as follows. We say that observer $b$ is behind observer $k$ iff

$$
\begin{aligned}
\forall m \in \text { IOb } \forall t \in D o m T r_{m}^{k} \forall \vec{p}, \vec{q} \in C d_{m} \quad \vec{p} \in t r_{m}(k) \wedge \\
\vec{q} \in t r_{m}(b) \wedge e v_{m}(\vec{p}) \sim_{k}^{\mu} e v_{m}(\vec{q}) \wedge \vec{a}_{m}^{k}(t) \uparrow \uparrow(\vec{p}-\vec{q}) .
\end{aligned}
$$

The following theorem shows that if observer $b$ is at a lower level in the tower than observer $k$ is, then his clock runs slower than the clock of $k$, as seen by $k$ with radar.

Theorem 5.8. Assume AccRel ${ }_{d}, \mathrm{~A} \times \exists \mathrm{Ob}$ and $d \geq 3$. Let $b, k \in \mathrm{Ob}$ such that:

(1) $k$ is positively accelerated,

(2) $b$ and $k$ are coplanar,

(3) $b$ is behind $k$.

Then the clock of $k$ runs slower than the clock of $b$, as seen by $k$ with radar.

The proofs, along with more explanation and motivation, of the theorems presented in this section can be found in 45 .

\section{Questions, SUGGESTIONS FOR FUtURE RESEARCH}

(1) We hope that the perspective outlined in sections [13, and the techniques presented in sections [45 [44 already suggest a research proposal. Sections 4 [5 cover only a small fragment of the research proposed in sections [1] So the proposal is: elaborate a larger part of the perspective outlined in 013 in the style of sections 0 (15] and [44.

(2) The Introduction of 2] contains more ideas both on the general perspective (of applying logic to spacetime theory) and also more of the long-distance goals. However, some of the present results were not available when [2] was written, therefore that introduction does not replace completely the present section.

(3) In section 5 we started to elaborate a purely logical theory of the effects of gravitation on clocks. Elaborate this direction in more detail, and investigate more aspects of gravitation on clocks. E.g. assume we bore a hole through the Earth from the North pole to the South pole. Now put a clock into the middle of the Earth. It will levitate "weightlessly" in the middle. Put another clock to the surface of the Earth. It will be squeezed by gravity to the surface. Despite this, 
the clock levitating in the middle will run slower than the one on the surface. A third clock high above in deep space will run even faster (than the one on the surface). Why? Find a logic style formulation of the above (and prove it) in the manner of section 5 .

(4) Investigate/formulate further aspects of the effects of gravity on instruments (like clocks, meter-rods). E.g. define the so-called gravitational force-field experienced by an accelerated observer (via acceleration, relative to the observer, of test particles dropped by the observer). Study this force-field and connect this study with the investigations in section 5. Try to make an integrated coherent picture of gravity, time warp (clock behavior in gravitational fields), and gravitational force. (Remark: gravitational force is often suppressed in the literature because it is not "absolute", i.e. is not observer independent. All the same, if we keep in mind that it is observer dependent, then it is a helpful concept.) Imagine a long, accelerated spaceship. The gravitational force experienced in the aft of the ship will be greater than that in the front of the ship. Why?

(5) Continuing in the spirit of sections 4,5, 44, and the above, elaborate a FOL theory of the spacetime of a Schwarzschild black hole 63. Streamline that theory, make it logically transparent and illuminating. Apply conceptual analysis to the theory similar in spirit as conceptual analysis of special relativity is started in [2, 1, 43. Using the theory of accelerated observers and Einstein's equivalence principle, create a logically convincing, illuminating theory of such black holes. In this direction it might be helpful that the analogy between the world-view or reference frame of an accelerated spaceship and skyscrapers (towers) on the event horizon of a black hole is described in detail in Rindler's relativity book [52, §12.4 "The uniformly accelerated lattice", pp.267272]. Figure 12.6 is especially useful therein. Also note how in Rindler's arrangement of the skyscrapers above the black hole they are prevented from falling by rigid rods separating them (these rods provide the "acceleration" experienced by the inhabitants of the towers/spaceships). These rods are called struts in [52, p.270, line 7 bottom up].

So, we suggest combining the presently started FOL theory of accelerated observers and of effects of gravity (acceleration) on instruments of observers with the just quoted part of Rindler's work in order to elaborate a FOL theory of the simplest kind of black holes. Of course, the main point is that we are striving for a very special kind of illuminating (etc.) FOL theory (and not just any FOL theory describing a black hole).

When the above is done, we suggest applying re-coordinatization in order to obtain an Eddington-Finkelstein version of this FOL theory of the black hole. This second (EF) version of the theory will also describe what the in-falling observer sees e.g. from inside the event horizon. For the latter question we suggest assuming that the black hole is huge 
(galactic size) so that enough stuff remains to be observed after falling through the event horizon.

(6) After having streamlined, analyzed, simplified FOL theories of simple (but huge) black holes, we propose turning to what we call double black holes or exotic black holes. Double black holes have two event horizons, an outer one and an inner one. In theory and under certain assumptions, a traveler might fall into the black hole, survive this and may come out at some other point of spacetime (in our universe or in some other universe). So, some of these double black holes may be regarded kind of wormholes. Examples are spinning black holes (Kerr spacetime, Kerr-Newmann spacetime), and electrically charged black holes (Reissner-Nordström spacetime) 63 .

The task here is again to build up, streamline, and conceptually analyse, simplify FOL theories for such double black holes. They offer logically intriguing issues for the logician as indicated in section 3.

(7) Besides the relatively simple kind of acceleration studied in sections 4,5. 44, rotation provides a kind of acceleration appearing in the form of the centrifugal force. A further research task is to analyse via FOL the world-view represented by a rotating coordinate lattice (relative to the gyroscopes) and generally, the rotational spacetimes. An example for these is the slowly rotating black hole (Kerr spacedtime), other examples are Gödel's rotating universe, Tipler van Stockum spacetime. In these spacetimes rotation leads to CTC's and to many other exotic effects like the so-called dragging of inertial frames or the drag effect. Finding out more about these is the task of NASA's recent "Probe B". Here again a FOL theory of such spacetimes waits for the creation, conceptual analysis and detailed illuminating explanation of what happens and exactly why. A particular question waiting to be answered is to find out and analyse what the common features/mechanisms/principles of these rotating spacetimes (with CTC's) are. E.g., many features of the above mentioned three spacetimes coincide. Is this a coincidence or is there a more general "theory of rotating spacetimes" lurking in the background. For more on this question we refer to [5]. In particular, we are looking for a logical answer to the quasi-philosophical question: "Exactly why and how CTC's are generated in rotating black holes and in Gödel's universe. Why do they counter-rotate with matter?" (More on what we call "counter-rotation" can be found in [5].)

\section{ACKNOWLEDGEMENTS}

Thanks go to Victor Pambuccian for many valuable conversations on the subject, for reading an earlier version of this paper and for helpful suggestions leading to the present version. 
Research supported by the Hungarian National Foundation for scientific research grant T43242 as well as by Bolyai Grant for Judit X. Madarász.

\section{REFERENCES}

[1] H. Andréka, J. X. Madarász and I. Németi, "Logical axiomatizations of space-time," In Non-Euclidean Geometries: János Bolyai Memorial Volume, A. Prékopa and E. Molnár eds. (Springer, Berlin, 2006), pp.155-185. http://www.math-inst.hu/pub/algebraic-logic/lstsamples.ps

[2] H. Andréka, J. X. Madarász and I. Németi, with contributions from A. Andai, G. Sági, I. Sain and Cs. Tóke, "On the logical structure of relativity theories," Research report, (Alfréd Rényi Institute of Mathematics, Budapest, 2002). http://www.math-inst.hu/pub/algebraic-logic/Contents.html

[3] H. Andréka, J. X. Madarász and I. Németi, "The Logic of Space-Time," in preparation.

[4] H. Andréka, J. X. Madarász and I. Németi, "Logical analysis of relativity theories," In First-order Logic Revisited (Logos, Berlin, 2004), pp.7-36.

[5] H. Andréka, I. Németi and C. Wüthrich, "A twist in the geometry of rotating black holes: seeking the cause of acausality," in preparation.

[6] J. Ax, "The elementary foundations of spacetime," Found. Phys. 8, 507 (1978).

[7] J. Barwise and J. Etchemendy, The Liar: An essay on truth and circularity (Oxford University Press, New York, 1987).

[8] C. H. Brans, "Gravity and the Tenacious Scalar Field," (1979). arXiv $/ \mathrm{gr}-\mathrm{qc} / 9705069$

[9] C. H. Brans, "The roots of scalar-tensor theory: an approximate history," (2004). arXiv gr-qc/0506063

[10] S. M. Carroll, "Why is the Universe Accelerating," (2003). arXiv astro-ph/0310342

[11] C. C. Chang and H. J. Keisler, Model Theory (North-Holland, Amsterdam, 1973, 1990).

[12] R. d'Inverno, Introducing Einstein's Relativity (Clarendon, Oxford, 1992).

[13] Gy. Dávid, "Modern cosmology - astronomical, physical and logical approaches," Abstracts of Invited talks in "Logic in Hungary 2005". http://atlas-conferences.com/cgi-bin/abstract/caqb-64 Transparencies of the talk: http://www.logicart.hu/events/lh05/index.php (in the Invited Speakers section)

[14] Gy. Dávid and I. Németi, "Relativistic Computers and the Turing Barrier," To appear in J. Appl. Math. Comput., (2006). http://renyi.hu/pub/algebraic-logic/beyondturing.pdf

[15] J. Earman, Bangs, Crunches, Whimpers, and Shrieks (Oxford Univ., New York, 1995).

[16] J. Earman and J. D. Norton, "Forever Is a Day: Supertasks in Pitowsky and Malament-Hogarth Spacetimes," Philos. Sci. 60, 22 (1993).

[17] J. Earman, C. Smeenk, and C. Wüthrich, "Take a Ride on a Time Machine," In R. Jones and P. Ehrlich, eds., Reverberations of the Shaky Game: Festschrift for Arthur Fine (Oxford Univ., Oxford, forthcoming).

[18] A. Einstein, Über die spezielle und die allgemeine Relativitätstheorie (von F. Vieweg, Braunschweig, 1921).

[19] G. Etesi and I. Németi, "Non-turing computations via Malament-Hogarth space-times," Int. J. Theor. Phys. 41, 341 (2002). arXiv gr-qc/0104023 
[20] V. Faroni, "Illusions of general relativity in Brans-Dicke gravity," Phys. Rev. D 20, 084021 (1999).

[21] J. Ferreirós, "The road to modern logic - an interpretation," B. Symb. Log. 7, 441 (2001).

[22] H. Friedman, On foundational thinking 1, Posting in FOM (Foundations of Mathematics) Archives www.cs.nyu.edu (January 20, 2004).

[23] H. Friedman, On foundations of special relativistic kinematics 1, Posting No 206 in FOM (Foundations of Mathematics) Archives www.cs.nyu.edu (January 21, 2004).

[24] L. Fuchs, Partially Ordered Algebraic Systems (Pergamon, Oxford, 1963).

[25] K. Gödel, "An Example of a New Type of Cosmological Solution of Einstein's Field Equations of Gravitation," Rev. Mod. Phys., 21, 447 (1949).

[26] R. Goldblatt, "Orthogonality and spacetime geometry," (Springer-Verlag, New York, 1987).

[27] R. J. Gott, "Closed timelike curves produced by pairs of moving cosmic strings: Exact solutions," Phys. Rev. Lett. 66, 1126-1129 (1991).

[28] R. J. Gott, Time Travel in Einstein's Universe: The Physical Possibilities of Travel Through Time (Houghton Mifflin, New York, 2002).

[29] A. Günbaum, Philosophical Problems of Space and Time (Dover, New York, 1963).

[30] P. Hájek and P. Pudlák, Metamathematics of first-order arithmetic (Springer, 1993).

[31] S. W. Hawking and G. F. R. Ellis, The Large Scale Structure of Space-Time (Cambridge Univ., Cambridge, 1973).

[32] L. Henkin, A. Tarski and P. Suppes (eds.) The Axiomatic Method with Special Reference to Geometry and Physics (North-Holland, Amsterdam, 1959).

[33] D. Hilbert, "Mathematische Behandlung der Axiome der Physik," Akad. Wiss. Gttingen 272-273 (1900). http://www.mathematik.uni-bielefeld.de kersten/hilbert/prob6.html

[34] D. Hilbert, "Uber den Satz von der Gleichheit der Basiswinkel im gleichschenkligen Dreieck," Proc. London Math. Soc. 35, 50 (1902/1903).

[35] R. Hirsch, "Dialectics and Logic," Cultural Logic (Spring 2004).

[36] W. Hodges, Model Theory (Cambridge Univ., Cambridge, 1997).

[37] M. Hogarth, "Deciding arithmetic using SAD computers," Brit. J. Phil. Sci. $\mathbf{5 5}, 681(2004)$.

[38] M. L. Hogarth, "Predictability, Computability and Spacetime," PhD thesis, (Univ. of Cambridge, UK, 2002). http://www.renyi.hu/pub/algebraic-logic/Hogarththesis.ps.gz

[39] R. Horváth, "An Alexandrov-Zeeman type theorem and relativity theory," TDK paper, (Eötvös Loránd Univ., Budapest, 2005).

[40] R. Horváth and G. Székely, "An Alexandrov-Zeeman type theorem and its consequences in special relativity," in preparation.

[41] H. A. Lorentz "Electromagnetic phenomena in a system moving with any velocity less than that of light," Proc. Royal Acad. Amsterdam 6, 809 (1904).

[42] J. X. Madarász, Logic and relativity (in the light of definability theory), PhD thesis, (Eötvös Loránd Univ., Budapest, 2002). http://www.math-inst.hu/pub/algebraic-logic/Contents.html

[43] J. X. Madarász, I. Németi and Cs. Töke, "On generalizing the logic-approach to space-time towards general relativity: first steps," In First-order Logic Revisited (Logos, Berlin, 2004). pp.225-268. 
[44] J. X. Madarász, I. Németi and G. Székely, "Twin Paradox and the Logical Foundation of Relativity Theory," To appear in Found. Phys. (2006). arXiv gr-qc/0504118.

[45] J. X. Madarász, I. Németi and G. Székely, "A logical analysis of the time-warp effect of general relativity," in preparation.

[46] C. W. Misner, K. S. Thorne and J. A. Wheeler, Gravitation (W.H. Freeman, San Francisco, 1973).

[47] B. O'Neill, The Geometry of Kerr Black Holes (A K Peters, Massachusetts, 1995).

[48] V. Pambuccian, "Axiomatizations of hyperbolic and absolute geometries," In Non-Euclidean Geometries: János Bolyai Memorial Volume, A. Prékopa and E. Molnár, eds. (Springer, Berlin, 2006). pp.119-153.

[49] C. S. Reynolds, L. W. Brenneman and D. Garofalo, "Black Hole Spin in AGN and GBHCs," Astrophys. Space Sci. 300, 71 (2005). arXiv astro-ph/0410116

[50] H. Reichenbach, Axiomatik der relativistische Raum-Zeit-Lehre (1924), English translation:Axiomatization of the theory of relativity (Univ. of California, Berkeley, 1969).

[51] H. Reichenbach, The Philosophy of Space and Time (Dover, New York, 1958).

[52] W. Rindler, Relativity. Special, General and Cosmological (Oxford University Press, 2001).

[53] W. Rudin, Principles of Mathematical Analysis (McGraw-Hill, New York, 1953).

[54] J. Q. Schutz, Foundations of Special Relativity: Kinematic Axioms for Minkowski Space-Time (Springer, Berlin, 1973).

[55] Gy. Serény, "Boolos-style proofs of limitative theorems," Math. Log. Quart 50,2 211 (2004).

[56] S. G. Simpson, (editor), Reverse Mathematics 2001, Lecture Notes in Logic, Association for Symbolic Logic, 2005, X + 401 pages.

[57] W. J. van Stockum, "The gravitational field of a distribution of particles rotating around an axis of symmetry," Proc. Roy. Soc. Edinburgh A 57, 135 (1937).

[58] T. E. Strohmayer, "Discovery of a $450 \mathrm{HZ}$ Quasi-periodic Oscillation from the Microquasar GRO J1655-40 with the Rossi X-Ray Timing Explorer," Astrophys. J. Lett., 552, L49 (2001). arXiv astro-ph/0104487

[59] P. Suppes, "Axioms for relativistic kinematics with or without parity," In L. Henkin, P. Suppes and A. Tarski (eds.), The Axiomatic Method with Special Reference to Geometry and Physics (North-Holland, Amsterdam, 1959).

[60] P. Suppes, "The desirability of formalization in science," J. Philos. 65, 651 (1968).

[61] G. Székely, A first-order logic investigation of the twin paradox and related subjects, Master's thesis, Eötvös Loránd Univ., Budapest (2004).

[62] A. Tarski, A Decision Method for Elementary Algebra and Geometry (Univ. of California, Berkeley, 1951).

[63] E. F. Taylor and J. A. Wheeler, Exploring Black Holes: Introduction to General Relativity (Addison Wesley, San Francisco, 2000).

[64] F. J. Tipler, "Rotating Cylinders and the Possibility of Global Causality Violation," Phys. Rev. D 9, 2203 (1974).

[65] J. Väänänen, "Second-order logic and foundations of mathematics," B. Symb. Log. 7, 504 (2001).

[66] R. M. Wald, General Relativity (Univ. of Chicago, Chicago, 1984).

[67] J. Woleński, "First-order logic: (philosophical) pro and contra," In First-Order Logic Revisited (Logos, Berlin, 2004). 